hep-th/9710178

LBNL-40889

RU-97-85

UCB-PTH-97/51

\title{
Why Matrix Theory is Hard
}

\author{
Michael R. Douglas ${ }^{1,2}$ and Hirosi Ooguri ${ }^{3,4}$ \\ ${ }^{1}$ Institut des Hautes Études Scientifiques \\ Le Bois-Marie, Bures-sur-Yvette, 91440 France \\ 2 Department of Physics and Astronomy \\ Rutgers University, Piscataway, NJ 08855-0849 \\ ${ }^{3} 366$ Le Conte Hall, Department of Physics \\ University of California, Berkeley, CA 94720-7300 \\ ${ }^{4}$ Theory Group, Mail Stop 50A-5101, Physics Division \\ Lawrence Berkeley National Laboratory, Berkeley, CA 94720 \\ douglas@ihes.fr, ooguri@thsrv.lbl.gov
}

Recently Sen and Seiberg gave a prescription for constructing the matrix theory in any superstring background. We use their prescription to test the finite $N$ matrix theory conjecture on an ALE space. Based on our earlier work with Shenker, we find a sharper discrepancy between matrix theory computation and supergravity prediction. We discuss subtleties in the light-front quantization which may lead to a resolution to the discrepancy.

October 1997 


\section{Introduction}

Matrix theory [1] has by now passed a number of significant tests in toroidal backgrounds, which preserve maximal supersymmetry [2].

Its status in curved backgrounds is less clear. Although definitions have been proposed, which make contact with existing results in superstring duality, evidence that they correctly describe dynamics not constrained by supersymmetry or BPS arguments is still lacking. It is also not yet clear to what extent the large $N$ limit of the original proposal is a necessary ingredient, or whether finite $N$ gauge theory also has a Matrix theory interpretation, Discretized Light Cone Quantization (DLCQ [3, [1]) M theory, as proposed by Susskind [5]. In particular, a result which appears to contradict this (which we will review in detail shortly) was found in studying compactifications on K3 [6]: the definition obtained using the weak string coupling limit of D0-branes does not reproduce the expected interactions between gravitons; furthermore it is very difficult to do this using any quantum mechanics of finitely many degrees of freedom as a definition.

The study of toroidal compactification (as reviewed in [2]) has shown that additional light states can be relevant after compactification and this raised a possibility that these might modify the conclusions of [6]. Recently significant light was shed on this issue by Sen [7] and Seiberg [8] (see also [9,10]), who showed that all existing definitions of Matrix theory including DLCQ can be obtained by carefully following the BFSS prescription, starting with theories of D0-branes in Ila superstring theory, boosting to the IMF and taking the limit of weak string coupling. Seiberg's argument is that this limit should be thought of as turning the boosted space-like hidden $S^{1}$ of IIa superstring theory into the null $S^{1}$ of DLCQ M theory. We find the simplicity of this argument compelling.

This prescription naturally generalizes to any superstring background and for the large volume limit of $\mathrm{K} 3$, it leads directly to the definition studied in [6]; we show in section 2 that additional states do not contribute. In section 3 we verify the claims of [6] in the regime of large K3 volume, large distances and large curvature lengths. This sharpens the contradiction considerably.

We discuss possible resolutions in section 4. One possibility is that the computation

of [6] should not be compared with classical supergravity results; corrections in DLCQ $\mathrm{M}$ theory are important. However, assuming distances and curvature lengths are large compared to the eleven-dimensional Planck scale, we did not find candidates.

Rather, we will suggest as a possible resolution the idea that there is a subtlety in the argument of [8], which appears in taking the limit in which the space-like circle becomes 
null. Doing this step explicitly would require integrating out modes of zero longitudinal momentum, and in the analogous study of light-front gauge theory it is known that this generally produces modifications of the Hamiltonian. This picture could be tested by checking that there exist modified Hamiltonians which reproduce predictions of DLCQ M theory.

\section{M theory in curved space}

Recently a precise proposal for Matrix theory in a wide class of backgrounds, with any amount of supersymmetry, was formulated in [7,8]. Here we will show that, in the case of compactification on K3, their proposal leads directly to the definition studied in [6].

According to [7,8], M theory with Planck scale $m_{p}$ compactified on a light-like circle of radius $R$ and momentum $p_{-}=\frac{N}{R}$ is equivalent to $\widetilde{M}$ theory, which is Ila string theory with Planck scale $\widetilde{m}_{p}$ compactified on a space-like circle of radius $\widetilde{R}_{s}$ with $N$ D0-branes in the limit,

$$
\widetilde{R}_{s} \rightarrow 0, \quad \widetilde{m}_{p} \rightarrow \infty
$$

while keeping

$$
\widetilde{R}_{s} \widetilde{m}_{p}^{2}=R m_{p}^{2}
$$

finite. Any transverse length scale $l$ (distance between points, compactification radius, etc.) is rescaled to the length $\widetilde{l}$ in the $\widetilde{M}$ theory defined by

$$
\widetilde{l} \widetilde{m}_{p}=l m_{p}
$$

Let $\widetilde{g}_{s}$ and $\widetilde{l}_{s}=1 / \widetilde{m}_{s}$ be the string coupling constant and the string length of $\widetilde{M}$ theory. The limit takes

$$
\begin{aligned}
\widetilde{m}_{s}^{2} & =\widetilde{R}_{s} \widetilde{m}_{p}^{3} \rightarrow \infty \\
\widetilde{g}_{s} & =\left(\widetilde{R}_{s} \widetilde{m}_{p}\right)^{3 / 2} \rightarrow 0 \\
\widetilde{l} & =\widetilde{g}_{s}^{1 / 3} \operatorname{lm}_{p} \rightarrow 0
\end{aligned}
$$

and thus, if we do not make further $\mathrm{U}$ dualities, $\widetilde{M}$ theory is perturbative string theory in the substringy regime studied in [12]. 
Let us review some facts about this regime. The rescaling (2.4) sends the string tension to infinity and at first sight this might seem to be the usual low energy limit of string theory. If this were the case we could simply rescale the effective action

$$
S=\frac{1}{\widetilde{g}_{s}^{2} \widetilde{l}_{s}^{8}} \int d^{10} x \sqrt{g}\left(R+\zeta(3) \widetilde{l}_{s}^{6} R^{4}+c \widetilde{g}_{s}^{2} \widetilde{l}_{s}^{6} R^{4}+\ldots\right)
$$

using (2.4) to obtain the effective action of M theory. However, attempting this rescaling produces

$$
S=\frac{R}{l_{p}^{8}} \int d^{10} x \sqrt{g}\left(R+\zeta(3) \frac{l_{p}^{6}}{\widetilde{g}_{s}^{2}} R^{4}+c l_{p}^{6} R^{4}+\ldots\right)
$$

and we see that the $O\left(l_{s}\right)$ corrections to the action dominate the M theory terms.

The resolution of this problem is to recognize that substringy D-brane physics is not defined by closed string theory or its supergravity limit but is instead defined by open string theory. Indeed, very general world-sheet arguments given in detail at leading order and outlined at all orders in [12] show that the leading terms in (2.5) which survive the limit (2.4) are described by the theory of the lightest open strings. The mass of the lightest open string state stretching between two D-branes at finite distance $d$ is given by

$$
\widetilde{d} \widetilde{m}_{s}^{2}=d R m_{p}^{3}
$$

and thus remains finite, if we do not take the $R \rightarrow \infty$ limit of [1]. On the other hand, the gap to the first excited open string state goes as $\sqrt{\left(d R m_{p}^{3}\right)^{2}+\widetilde{m}_{s}^{2}}-d R m_{p}^{3} \sim \widetilde{m}_{s}$ at finite $R$ and hence diverges.

Thus $\widetilde{M}$ theory on very general grounds is an open string theory, and because of this many conventional expectations for $\mathrm{M}$ theory are not manifest in the matrix description. Even the $\sqrt{g} R$ term in (2.6) cannot be taken for granted, because other terms appear to dominate it in the limit. An example of this is the case of the $\mathbb{C}^{3} / \Gamma$ orbifold target space [13], where it is argued that the gauge theory construction does not naturally produce a Ricci flat metric.

A feature of $\widetilde{M}$ theory not addressed in [12] is the fact that the overall size of a compact target space $\mathcal{M}$ will also shrink to zero in the limit (2.4). In general this leads to additional light states, both open strings winding about $\mathcal{M}$ and D-branes wrapping cycles in $\mathcal{M}$. In small radius limits, general expectations from superstring duality and much work on toroidal compactification show that the wrapped D-brane states can be as important as or dominate the open string states. 
On the other hand, we will now argue that for a four dimensional compact target $\mathcal{M}$, in the infinite volume limit (in the original $\mathrm{M}$ theory sense), these states are unimportant. In conventional treatments of gravity (in space-time dimension greater than three) this limit is simple to take (there are no significant IR divergences), but given that gravity is being derived in this framework we must check this point explicitly.

We assume that $\mathcal{M}$ is smooth with a volume $V \equiv L^{4}$ and characteristic curvature length $l_{C}$. It may also have non-trivial $p$-cycles with volumes $V_{p}$; generically $V_{p} \sim l_{C}^{p}$.

The BPS states associated with compactness are wrapped $\mathrm{D} p$-branes, with mass of order

$$
\frac{\widetilde{V}_{p}}{\widetilde{g}_{s} \widetilde{l}_{s}^{p+1}}=\widetilde{g}_{s}^{(p-4) / 3} V_{p} R m_{p}^{p+2}
$$

which is infinite for $p<4$ and $g_{s} \rightarrow 0$. The D4-brane wrapping on $\mathcal{M}$ itself has mass of order $L^{4} R m_{p}^{6}$ and winding strings have masses $L R m_{p}^{3}$. These states become arbitrarily massive in the infinite volume limit in M theory, even though the $\widetilde{M}$ theory is compactified on a manifold shrinking to zero size. Thus we justify the reduction to quantum mechanics in this case. For D0 branes on a resolved ALE space, the gauge theory of [14] would describe such a system.

This would break down for compactification on a six-dimensional manifold, as noted in [7,8], and it is not clear whether the large volume limit has associated subtleties.

\section{3. $\widetilde{M}$ theory as a naive limit of string theory}

In this section we follow section 2 of [6] and make the assumption that the $\widetilde{M}$ theory action should be exactly given by the result of the procedure of section 2 . We review the computation of the leading supergravity interaction, a non-BPS result, and show that it does not agree with classical expectations. We compute the $v^{4}$ term in the scattering amplitude of two gravitons on $R^{5,1} \times \mathcal{M}$. The gravitons are fixed at points in $\mathcal{M}$ but move on $R^{5,1}$ with relative velocity $v$ and impact parameter $b$.

We first comment that for the result to have any chance to agree with supergravity, the mass of the lightest open strings must be equal to $\widetilde{\sigma}\left(x_{1}, x_{2}\right) \tilde{m}_{s}^{2}=\sigma\left(x_{1}, x_{2}\right) R m_{p}^{3}$, where $\sigma\left(x_{1}, x_{2}\right)$ is the geodesic distance between the two points $x_{1}$ and $x_{2}$ on $\mathcal{M}$. As found in the examples in [6] and from the discussion in [15, 16, 17], this is a highly non-trivial constraint on the non-linear sigma model, and it is not at all obvious from the weak coupling string theory point of view that it should be satisfied. It should be noted that the sigma-model on 
the string worldsheet is strongly coupled in the regime $\widetilde{\sigma}, \widetilde{l}_{C} \ll \widetilde{l}_{s}$. We have no results for or against this point in the present problem, so in the following we will make the assumption that it is satisfied, but it could equally well be false, leading to immediate disagreement with the classical supergravity limit of $M$ theory.

The $v^{4}$ term at the open string one-loop on K3 has the following general structure [6].

$$
\mathcal{A}_{v^{4}} \simeq \int_{0}^{\infty} d t t^{5 / 2} \exp \left(-t\left(\widetilde{b}^{2}+\widetilde{\sigma}^{2}\left(x_{1}, x_{2}\right)\right) \widetilde{m}_{s}^{2}\right) g(t)
$$

Here

$$
g(t)=\sum_{i=0}^{\infty} C_{i} e^{-h_{i} t}
$$

is related to the multiplicities of the $N=4$ superconformal algebra representations on the open string worldsheet, with $h_{0}=0$ corresponds to the lightest state of the open string. We can then perform the integral and find, using (2.4),

$$
\mathcal{A}_{v^{4}} \propto \sum_{i} \frac{C_{i}}{\left(b^{2}+\sigma^{2}\left(x_{1}, x_{2}\right)+\widetilde{g}_{s}^{-2 / 3} l_{p}^{2} h_{i}\right)^{7 / 2}} .
$$

We now see that in the $\widetilde{g}_{s} \rightarrow 0$ limit, the contribution from the massive excitations disappears provided the gap $\min _{i \geq 1}\left(h_{i}-h_{0}\right)$ of CFT spectrum remains finite in the limit. This is not something that should be taken for granted as the gap may disappear when CFT becomes singular [18, 19,20]. In our case, however, it is easy to see that this is generically true even in the substringy regime $\widetilde{\sigma}, \widetilde{l}_{C} \ll \widetilde{l}_{s}$. First of all, in the orbifold limit with non-zero theta-angle, we can compute the spectrum explicitly and show that the gap is finite. We can resolve the singularity by turning on the marginal perturbation of CFT (corresponding to adding the Fayet-Illiopoulos term to the D0 brane action [14]), and the gap does not disappear under the small perturbation. Therefore the massive excitations decouple in the limit $\tilde{g}_{s} \rightarrow 0$, confirming the claim in section 2 .

In this limit, we find

$$
\mathcal{A}_{v^{4}} \rightarrow \frac{C_{0}}{\left(b^{2}+\sigma^{2}\left(x_{1}, x_{2}\right)\right)^{7 / 2}}
$$

We can compare this with the supergravity prediction [6]. By keeping $x_{1}$ and $x_{2}$ sufficiently far away from the orbifold point, we can set the distance between the points $\sigma\left(x_{1}, x_{2}\right)$ to be smaller than the curvature radius $l_{C}$ measured near these points. We can then use the short distance expansion of the gravition scattering amplitude and compare it with (3.4). We find that subleading terms in the expansion are missing here. As pointed out in [6], the 
discrepancy remains even if there are corrections to the mass of the lightest open string state, as long as the massive excitations decouple and we are left with the gauge theory degrees of freedom.

Now at large $N$ it is possible that the subleading correction to (3.4) will appear as bound state effects, but for $N_{1}=N_{2}=1$ this is not possible.

We note that the same problem can be studied in other curved backgrounds, and the general properties of gauge theory and supergravity are so different as to make universal agreement quite unexpected. As an example, the dynamics of D0-branes in a five-brane background can be studied, and in this case even the relation $m_{W}=\widetilde{\sigma}\left(x_{1}, x_{2}\right) \widetilde{m}_{s}^{2}$ appears to fail [21].

\section{Possible resolutions}

The computation we described is valid at finite $N$ and thus the result should be compared with DLCQ supergravity. Could this interaction be different?

The first guess one might try is that supergravity loop effects are important. On physical grounds this requires some energy or momentum in the problem comparable to $m_{p}$, while we find disagreement even in the case of all length scales large compared to $l_{p}$.

The only new feature of DLCQ is the quantization $p_{-}=N / R$ and from this point of view $N$ small is advantageous. We should take the conventional parameter $R \gg m_{p}^{-1}$. We also require $m_{p} \gg p_{+}=p_{\perp}^{2} / 2 p_{-}$and here $N$ small means that we must have $p_{\perp}^{2} \ll m_{p}^{2}$ to have both $p_{+} \ll m_{p}$ and $p_{-} \ll m_{p}$. The resulting uncertainty relation $\Delta x \sim 1 / p$ leads to no further restrictions. We conclude that the usual energy suppression of loop effects in gravity is still valid in DLCQ.

Even on the classical level, DLCQ supergravity has not been much studied in the past, with some exceptions [22], and so it is conceivable that there are other subtleties relevant for this problem.

We finally come to our suggested resolution. The assumption made in section 3 that Matrix theory can be obtained by a naive application of the limiting procedure of [7,8] leads to contradictions with the expected physics of $M$ theory.

Does this mean that Matrix theory is wrong? Not necessarily, but it appears to mean that the boost to the infinite momentum frame is complicated.

Evidence for this idea can be found in the study of the analogous case of light-front quantization of gauge theory. (Some representative recent work can be found in [23].) This 
has been much studied over the years, especially as a non-perturbative approach to QCD, and has numerous advantages over the equal-time canonical approach. For example, in a theory without massless particles, the perturbative vacuum is the Fock vacuum - the complicated problem of solving for the vacuum state is eliminated.

These advantages do not come for free and the loss of Lorentz invariance means that the problem of renormalization is much more complicated. In particular, modes with longitudinal momentum $p_{-}=0$ must be integrated out to derive such a canonical formulation, and this leads to non-local divergences and counterterms which can only be determined by requiring Lorentz invariance in the resulting physics.

We can compare this with the discussion in [17]. There it was argued based on the results of [6, 13] and [12] that although one can use D0-brane theories as definitions of Matrix theory, reproducing expectations from eleven-dimensional supergravity would require using a Lagrangian with renormalized parameters and possible additional interactions.

What consistency conditions determine the renormalized Lagrangian? We cannot impose eleven-dimensional Lorentz invariance in a general curved background. On the other hand, it is known that Lorentz invariance in flat space, the presence of spin two degrees of freedom, and very general consistency conditions lead to the Einstein action for the low energy physics of gravity [24]. Adding supersymmetry to this, we can ask as our substitute for Lorentz invariance that supergravity amplitudes be correctly reproduced starting with a non-singular Lagrangian. It was shown in [17,16 that this leads to very non-trivial constraints on the Lagrangian, which do admit solutions. (It should be pointed out that the conditions studied so far do not completely determine the matrix model action [16].)

Thus we suggest as the resolution of the paradox the idea that the boost to the infinite momentum frame renormalizes the Lagrangian in a way which will lead to correct eleven-dimensional physics.

Another consideration which is compatible with this idea is to study the inverse process of deriving a weak string coupling D0-brane Lagrangian from Matrix theory. The way to do this is outlined in [25]; they show that the $1+1$-dimensional gauge theory describing Matrix theory on $S^{1}$ renormalizes to a world-sheet theory of an arbitrary light-cone gauge strings, with the correct interaction. States with D0-branes are described as sectors with electric flux in the gauge theory, and they argue that in the renormalized theory strings will end on D0-branes in the appropriate way. 
One could follow the same approach for compactification on $S^{1} \times \mathcal{M}$ to derive the corresponding string theory compactification and D0-brane Lagrangian. The non-trivial renormalization in this process means that we can expect to get sensible world-sheet Lagrangians from a wide variety of starting points; there is no reason at all from this point of view why the starting Matrix theory Lagrangian and resulting D0-brane Lagrangian should be the same.

An interesting counterpart to the idea comes from considering further compactification on $T^{n} \times \mathcal{M}$. For $n>1$ and non-compact $\mathcal{M}$, this would lead to an $n+1$-dimensional supersymmetric non-linear sigma model, which is generally believed to be non-renormalizable.

Now that we believe that sensible interacting field theories in dimensions greater than four can exist, it seems premature to assume that such supersymmetric non-linear sigma models cannot be renormalizable. It might be that renormalizable models exist, and that this constraint will provide a more effective way of determining the correct Lagrangians.

To add to the mystery surrounding this question, however, we must note that for $\mathcal{M}_{4}$ compact and $n>1$, as we mentioned, it could be that the large volume limit is subtle. This comment might also be relevant to the contradiction that supergravity predicts that six dimensional Calabi-Yau manifolds with $R l_{p}^{2} \ll 1$ will be Ricci flat, while weak coupling string theory with $R l_{p}^{2} \ll 1 \ll R l_{s}^{2}$ predicts that D0-branes do not see a Ricci flat metric [13,26], which otherwise would be direct evidence for our proposal.

However, these subtleties do not enter for the case of K3 compactification, and our overall conclusion is that the Matrix theory Lagrangian must be different from the weak string coupling Lagrangian in order to reproduce the physics of $M$ theory.

A priori the relation between these two Lagrangians is likely to be quite complicated, and this is the meaning of the title of the paper. Of course the Matrix theory has already provided simpler arguments for duality as well as much insight; however these issues will have to be addressed to get non-BPS results.

\section{Note added}

After the completion of this work we received a paper [27], where they found a discrepancy between the three graviton scattering amplitude calculated from the matrix theory and the supergravity prediction. As we are studying the two graviton scattering in a weak gravity background, there may be a relation between the results. A strong test of the idea described here will be to find a modified maximally supersymmetric Lagrangian which fixes this problem. 


\section{Acknowledgements}

We would like to thank Tom Banks, Kiyoshi Higashijima, Ashoke Sen, Koichi Yamawaki and, in particular, Steve Shenker for discussion. H.O is supported in part by NSF grant PHY-951497 and DOE grant DE-AC03-76SF00098. 


\section{References}

[1] T. Banks, W. Fischler, S. H. Shenker and L. Susskind, "M Theory as a Matrix Model: a Conjecture," Phys. Rev. D55 (1997) 5112-5128; hep-th/9610043.

[2] T. Banks, "The State of Matrix Theory," talks given at SUSY '97 and Strings '97, hep-th/9706168.

[3] T. Maskawa and K. Yamawaki, "The Problem of $P^{+}=0$ mode in the Null Place Field Theory and Dirac's Method of Quantization," Prog. Theor. Phys. 56 (1976) 270.

[4] C. Pauli and S. Brodsky, "Discretized Light Cone Quantization: Solution to a Field Theory in One Space One Time Dimensions," Phys. Rev. D32 (1985) 2001.

[5] L. Susskind, "Another Conjecture about M(atrix) Theory," hep-th/9704080.

[6] M. R. Douglas, H. Ooguri and S. H. Shenker, "Issues in M(atrix) Theory Compactification," Phys. Lett. B402 (1997) 36-42; hep-th/9702203.

[7] A. Sen, "D0 Branes on $T^{n}$ and Matrix Theory," hep-th/9709220.

[8] N. Seiberg, "Why is the Matrix Model Correct?" hep-th/9710009.

[9] S. Hellerman and J. Polchinski, as referred to in [11.

[10] J. Maldacena, "Branes Probing Black Holes," talk presented at Strings 97; hepth/9709099.

[11] K. Becker, M. Becker, J. Polchinski and A. Tseytlin, "Higher Order Graviton Scattering in M(atrix) Theory," hep-th/9706072.

[12] M. R. Douglas, D. Kabat, P. Pouliot and S. Shenker, "D-branes and Short Distances in String Theory," Nucl. Phys. B485 (1997) 85-127; hep-th/9608024.

[13] M. R. Douglas and B. Greene, "Metrics on D-brane Orbifolds," hep-th/9707214.

[14] M. R. Douglas and G. Moore, "D-branes, Quivers, and ALE Instantons," hepth/9603167.

[15] M. R. Douglas, "D-branes in Curved Space," to appear in Adv. Theor. Math. Phys.; hep-th/9702048.

[16] M. R. Douglas, A. Kato and H. Ooguri, "D-brane Actions on Kähler Manifolds," to appear in Adv. Theor. Math. Phys.; hep-th/9708012.

[17] M. R. Douglas, "D-branes and Matrix Theory in Curved Space," talk given at Strings '97; hep-th/9707228.

[18] M. Bershadsky, S. Cecotti, H. Ooguri and C. Vafa, "Holomorphic Anomalies in Topological Field Theories," Nucl. Phys. B405 (1993) 279; hep-th/9302103.

[19] S. H. Shenker, "Another Length Scale in String Theory?" hep-th/9509132.

[20] H. Ooguri and C. Vafa, "Two-dimensional Black Hole and Singularity of CY Manifolds," Nucl. Phys. B463 (1996) 55; hep-th/9511164.

[21] D.-E. Diaconescu and M. R. Douglas, work in progress.

[22] M. Goroff and J. H. Schwarz, "D-Dimensional Gravity in the Light-Cone Gauge," Phys. Lett. 127B (1983) 61. 
[23] K. G. Wilson, T. Walhout, A. Harindranath, W. M. Zhang, R. J. Perry and S. Glazek, "Nonperturbative Light-Front QCD," Phys. Rev. D49 (1994) 6720-6766; hepth/9401153

G. McCartor, D. G. Robertson, S. S. Pinsky, "Vacuum Structure of Two-Dimensional Gauge Theories on the Light Front," Phys. Rev. D56 (1997) 1035-1049; hepth/9612083

S. Tsujimaru and K. Yamawaki, "Zero Mode and Symmetry Breaking on the Light Front," hep-th/9704171.

[24] S. Weinberg, "Photons and Gravitons in Perturbation Theory: Derivation of Maxwell's and Einstein's Equations," Phys. Rev. 138 (1965) B988; "Ultraviolet Divergences in Quantum Theories of Gravitation," in General Relativity, an Einstein Centenary Survey, ed. S. Hawking and W. Israel (1979, Cambridge University Press).

[25] R. Dijkgraaf, E. Verlinde, H. Verlinde, "Matrix String Theory," Nucl. Phys. B500 (1997) 43-61; hep-th/9703030.

[26] M. B. Green, M. Gutperle, P. Vanhove, "One Loop in Eleven Dimensions," hepth/9706175.

[27] M. Dine and A. Rajaraman, "Multigraviton Scattering in the Matrix Model," hepth/9710174. 